\title{
Another mile stone event for MOJ Clinical \& Medical Case Reports (MOJCR)
}

\section{Editorial}

MOJ Clinical \& Medical Case reports is an international, peer reviewed, open access journal. As we move forward it is with great pride that I announce to our readers that the Journal has been recognized for Indexing in ROAD. This represents another way that the Journal is recognized and its contents are made available to the scientific and medical communities. It is one more important form of recognition for the Journal. We continue to work to achieve an acceptance for indexing with additional indexing entities. The Journal is archived and available for all researchers to review.

The Journal is committed to providing a forum for the exchange of information regarding medical conditions which may prove valuable to wide spectrum of medical and clinical providers and researchers. To that end we strive to achieve the widest range of indexing to increase the availability of information across many specific fields. Manuscripts published in the Journal since its inception range from surgery to trauma, internal medicine, gastroenterology, orthopedics, ophthalmology and dental health.

One issue which often is asked is: Just what is meant by Open Access? The Journal's publisher has made this statement, available on the Journal's web site, to inform authors regarding the nature of "Open Access": "The Open Access Journals are meant to provide unrestricted access to all their content. They also levy no rules or restrictions on reusing the published articles in any kind. The global interested population and the scientific community can have Open Access to all the content."

"MedCrave provides you immediate access to all the scientific manuscripts for free. There are no rules set for the process of reusing content from here. The viewer can reuse the original works in an unrestricted way. You will not need any fee for membership or downloading. There are no legal issues either. You can reuse the entire original content here, you can copy the scientific research data and may distribute all the contemporary new technique to all, but on one condition - The original author of the manuscript and the original source of content must be clearly and properly cited."

Another area that is often questioned is the nature and role of Peer Review. Peer review is the use of independent experts to evaluate manuscripts which are submitted for publication. This system is a corner stone of medical and scientific publishing. Upon receipt the primary subject of the manuscript is determined, after which one or more members of the editorial board whose expertise is in the area of the case report which is under review. Reviewers are independent. The Editorial Board is made up of an international, very diverse, group of
Volume 8 Issue 4 - 2018

\author{
Stewart Michael Sharp \\ Editor-In-Chief, Regulatory Consulting, Inc, USA
}

Correspondence: S Michael Sharp, FRAPS, Regulatory Consulting, Inc, USA, Email drsmsharp@aol.com

Received: July 31, 2018 | Published: August 03, 2018

experts in all medical specialties.

That reviewer or reviewers will make a careful consideration of the manuscript. To help assure that there is consistency among reviewers. To help achieve that, as formal check-list of editorial criteria for publication has been developed and is used for all reviews:

1. The Manuscript Fits into the mission of the Journal

2. The abstract accurately reflects the content. The problem is significant and concisely stated

3. The experimental and/or theoretical methods are described comprehensively

4. The discussion interpretations and conclusions are justified by the result of the study

5. Adequate reference is made to other work in the field

6. The language, grammar and syntax are acceptable

7. Rate the priority of publishing the manuscript

Based upon the reviewer's recommendation the manuscript will either be accepted for publication and will begin the editorial publishing process. If the reviewer found areas of weakness that preclude the immediate publication of the manuscript it may be given to the Editor-In-Chief for additional review. If the Editor-In-Chief agrees the manuscript, along with comments and information as to the areas that require improvement will be returned to the author. If the author does not agree with the decision he/she may request an additional review. Such a review would be conducted by an independent member of the editorial board who did not participate in the initial review. It is our stated policy to provide high quality in the implementation of peer review, beginning with timely and efficient processing to expedite review and publication.

I want to thank and acknowledge those who are responsible for the growth of the Journal. This includes the publisher, our editorial Board, and, of course, our most important asset, the scientists and physicians who submit their manuscripts for publication. 